\section{Sequenced reinforcement in a}

$$
\text { two-way runway situation }
$$

\author{
ALLEN H. WOLACH, MAUREEN COUTTS, and MORRIS ADERMAN \\ Illinois Institute of Technology, Chicago, Ill. 60616
}

Capaldi's $(1963,1964)$ sequential response hypothesis was tested with a within-Ss experimental design. A two-way runway situation made it possible for $\mathrm{Ss}$ to experience one sequence of reinforcements and nonreinforcements while running in one direction and another sequence while running in the opposite direction. The direction in which Ss ran alternated from trial to trial. The sequences Ss experienced in the two directions were an $\mathrm{N}$-length of 3 (always 3 nonreinforced trials before a reinforced trial) or N-lengths of 1,2 , and 3 (either 1,2 , or 3 nonreinforcements before a reinforced trial). Greater resistance to extinction occurred in the direction that was associated with $\mathrm{N}$-lengths of 1 , 2 , and 3 .

A series of studies has been performed to determine the effects of the sequencing of nonreinforcements in a partial reinforcement situation (e.g., Capaldi et al, 1963; Capaldi, 1964). These studies have demonstrated that the number of nonreinforcements in a row ( $\mathrm{N}$-length) and number of different $\mathrm{N}$-lengths determine resistance to extinction. That is, Ss experiencing extended training with long $\mathrm{N}$-lengths during acquisition are more resistant to extinction than Ss with shorter $\mathrm{N}$-lengths. In addition, after extended training, partial reinforcement sequences with more than one $\mathrm{N}$-length produce greater resistance to extinction than single $\mathrm{N}$-length sequences if the maximum $\mathrm{N}$-length in the multiple $\mathrm{N}$-length sequence is the same as the single $\mathrm{N}$-length in the other sequence. For example, Capaldi (1964) demonstrated that Ss experiencing 120 trials with $\mathrm{N}$-lengths of 3,2 , and 1 in a $50 \%$ reinforcement situation were more resistant to extinction than $\mathrm{Ss}$ experiencing 120 trials with the $\mathrm{N}$-length of 3 in a $50 \%$ reinforcement situation. The relative resistance to extinction for the single and multiple $\mathrm{N}$-length groups is reversed when a small number of acquisition trials are used; e.g., Capaldi obtained greater resistance to extinction for the single $\mathrm{N}$-length group after 24 acquisition trials.

Capaldi hypothesized that Ss gain habit strength after experiencing the stimulus aftereffects of successive nonreinforcements followed by reinforcement. Every time an N-length is followed by reinforcement, habit strength builds up for that N-length. After extended training an asymptotic level of habit strength is attained. The Ss with extended training on a multiple N-length sequence have the same habit strength on the longest N-length as compared to Ss with that N-length as their only N-length. The multiple-N-length $S$ s also have habit strength on the shorter N-lengths.
Consequently, their total generalized habit of single-N-length Ss.

Less extensive training does not produce habit strength asymptote for $\mathrm{Ss}$ in the single- or multiple-N-length groups. Since $\mathrm{Ss}$ in the single-N-length group have more experiences with that $\mathrm{N}$-length than do the multiple-N-length Ss, the single-N-length Ss are closer to habit strength asymptote on the long N-length. Since long N-lengths produce greater resistance to extinction than shorter $\mathrm{N}$-lengths, Ss who are not near asymptote on their $\mathrm{N}$-lengths show greater resistance to extinction if all of their training is with the single-N-length sequence.

Capaldi's position has been tested in the context of between-group designs in which a given $\mathrm{S}$ experiences either the single- or multiple-N-length sequence. Partial reinforcement studies, moreover, have been performed with within-Ss designs. Hearst (1961) has shown that pigeons experiencing different percentages of reinforcement (VR-schedules) on two keys show greater resistance to extinction on the key that had been associated with the smaller percentage of reinforcement. If the sequencing of nonreinforcements during acquisition is the primary determinant of resistance to extinction, then it should be possible to show greater resistance to extinction after extended training for a multiple N-length sequence with a within-Ss design.

The present study was carried out in a two-way runway situation in which Ss alternated running direction from trial to trial. One direction was associated with a multiple $\mathrm{N}$-length reinforcement sequence and the other direction was associated with a single $\mathrm{N}$-length sequence.

$$
\text { SUBJECTS }
$$
Sprague-Dawley rats served as Ss for this investigation. The Ss were housed three to strength is greater than the habit strength

Fourteen naive 130 - to 135 -day-old male a cage with water available at all times. Three weeks prior to the initiation of preliminary training, Ss were placed on a 24-h feeding schedule. Eight $2-\mathrm{cm}$ Noyes pellets were placed in each cage at the same time every day. During preliminary training and the experiment proper, the eight pellets were placed in a given cage $5 \mathrm{~min}$ after the last rat in the cage finished its daily session. All of the rats in a cage were run prior to running the $S s$ in another cage. APPARATUS

The apparatus was a straight alley runway that had two equal-sized end compartments $(33.02 \times 12.70 \times 12.70 \mathrm{~cm})$. Two guillotine-type doors separated the end compartments from the center section of the runway. A third guillotine-type door divided the center section into two e $\mathrm{q} \mathrm{u}$ a 1 - $\mathrm{s}$ i z e d s e c $\mathrm{t}$ jon s $(69.85 \times 12.70 \times 12.70 \mathrm{~cm})$. The walls and floor of the runway were made of wood and painted flat black. Hinged frames with fine mesh aluminum screening served as tops for the center sections and the two end compartments.

\section{PROCEDURE}

Preliminary training consisted of $10-\mathrm{min}$ periods on 2 successive days in which an $S$ was allowed to explore the runway. Two $.045 \mathrm{-g}$ Noyes pellets were placed in the end compartment opposite to the one in which $S$ was placed. The side of the runway $S$ started in and the side of the runway the food was placed in were reversed on the second day of preliminary training.

During the experiment proper Ss experienced a two-way runway situation. A given $\mathrm{S}$ always started a daily session on the same side of the runway. When $S$ reached the opposite side of the runway, the guillotine-type door was closed behind it for $20 \mathrm{sec}$. After the $20-\mathrm{sec}$ period the door was raised and the $S$ was required to run to the end compartment that had been the startbox on the first trial. The Ss experienced 12 trials a day, 6 in each direction. Timing of the first trial was initiated when S's feet touched the starting end compartment floor. The trial was terminated when $S$ was $18 \mathrm{~cm}$ into the opposite end compartment and the guillotine-type door closed behind it. The remaining 11 trials were initiated by the raising of the guillotive-type door that confined $S$ in an end compartment and terminated when $S$ was $18 \mathrm{~cm}$ in to the opposite end compartment. The Ss were prevented from retracing their paths before the completion of a trial by means of the guillotine-type doors. If an $S$ failed to complete a trial in $90 \mathrm{sec}$, it was gently pushed to the appropriate end of the runway and confined in the end compartment for $20 \mathrm{sec}$

The Ss were divided randomly in to two 
Table 1

Reinforcement Sequences for Both Groups in the Two Running Directions and Independent of Direction

\begin{tabular}{|c|c|c|c|c|}
\hline & Days & Direction 1 & Direction 2 & Overall Sequence \\
\hline $\begin{array}{l}\text { Group } 1 \\
\text { (maximum } \\
\text { N-length } \\
\text { 3) }\end{array}$ & $\begin{array}{l}1,5,9,13,17 \\
2,6,10,14,18 \\
3,7,11,15,19 \\
4,8,12,16,20\end{array}$ & $\begin{array}{l}\text { NNNRRR } \\
\text { NNNRRR } \\
\text { NNNRRR } \\
\text { NNNRRR }\end{array}$ & $\begin{array}{l}\text { RRNNNR } \\
\text { RRNNNR } \\
\text { RNRNNR } \\
\text { RNRNNR }\end{array}$ & $\begin{array}{l}\text { NRNRNNRNRNRR } \\
\text { NRNRNNRNRNRR } \\
\text { NRNNNRRNRNRR } \\
\text { NRNNNRRNRNRR }\end{array}$ \\
\hline $\begin{array}{l}\text { Group } 2 \\
\text { (maximum } \\
\text { N-length } \\
6 \text { ) }\end{array}$ & $\begin{array}{l}1,5,9,13,17 \\
2,6,10,14,18 \\
3,7,11,15,19 \\
4,8,12,16,20\end{array}$ & $\begin{array}{l}\text { RNNNRR } \\
\text { RRNNNR } \\
\text { RNNNRR } \\
\text { RRNNNR }\end{array}$ & $\begin{array}{l}\text { RNNNRR } \\
\text { RRNNNR } \\
\text { NNRNRR } \\
\text { NRNNRR }\end{array}$ & $\begin{array}{l}\text { RRNNNNNNRRRR } \\
\text { RRRRNNNNNNRR } \\
\text { RNNNNRNNRRRR } \\
\text { RNRRNNNNNRRR }\end{array}$ \\
\hline
\end{tabular}

equal groups for the experiment proper, and each $S$ received 20 days of training. Table 1 shows the placement of the reinforced $(\mathrm{R})$ and nonreinforced $(\mathrm{N})$ trials for the two groups. A given $S$ experienced its sequence five times. Four of the Ss in each group started daily sessions at one end of the runway, and three Ss started at the other end. This table also shows the sequencing while running in a given direction. It can be seen that $S$ s always experienced three nonreinforcements in a row (N-lengths of 3 ) in one direction and 1,2 , or 3 nonreinforcements (N-lengths of 1,2 , or 3 ) in the other direction. For instance, on Day 1 each $S$ in the first group began with a nonreinforced trial (Direction 1) followed by a reinforced trial when it ran in the opposite direction (Direction 2), followed again by a nonreinforced trial when returning via Direction 1 (original direction), followed by another reinforced trial running in the second direction (Direction 2), etc. The overall sequence is illustrated in Table 1. The two groups differed in the maximum number of nonreinforcements in a row independent of direction. That is, even though both groups experienced $\mathrm{N}$-lengths of 3 in one direction and $\mathrm{N}$-lengths of 1,2 , and 3 in the other direction, the maximum number of nonreinforced trials without regard to direction was three for one group and six for the other group.

Eight days of extinction followed the 20 days of acquisition during which Ss experienced the same runway contingencies (12 extinction trials per day).

\section{RESULTS}

A repeated-measures analysis of variance (Running Direction by Experimental Group) was performed on the data from the last day of acquisition to ascertain if the $\mathrm{Ss}^{\prime}$ speeds differed in the two directions or if the two groups (maximum $\mathrm{N}$-length of 6 and maximum N-length of 3) differed. Each $S$ contributed two data points from its last day of acquisition. These data points consisted of the sum of the running speeds (1/latency in seconds) in the direction associated with the $\mathrm{N}$-length of 3 and the sum of the speeds in (id not from each other, $F(1,12)=.46, p>.05$. N-lengths of 6 or 3 .
The Experimental Group by Running Direction interaction was not significant, $F(1,12)=.00, p>05$

It is possible that the data from the first trial of each daily session are not comparable to the data from other trials because the $S$ was not handled before the subsequent trials. Hence, the data were reanalyzed after eliminating the first trial of each extinction day. The second trial of a daily session was also dropped from the analysis on alternate extinction days to insure that $S$ 's running speeds in the second running direction were not given undue weight. Thus, the data from an S's daily extinction session consisted of the sum of its last five trials in what had been the $\mathrm{N}$-length of 3 direction and the sum of all 6 , or the last five trials in the multiple $\mathrm{N}$-length direction. The two sums were divided by the number of trials composing them (five or six) and added to similarly corrected sums obtained from the remaining 7 days of extinction. Analysis of the "corrected" data produced the same results as the noncorrected data. The Ss were faster in the multiple N-length direction than in the single $\mathrm{N}$-length direction, $F(1,12)=9.08, p<.05$. The two experimental groups did not differ, $F(1,12)=.18, p>.05$. No difference was obtained for the Experimental Group by Running Direction interaction, $\mathrm{F}(1,12)=.09, \mathrm{p}>.05$.

\section{DISCUSSION}

The present study demonstrates that Capaldi's hypothesis can explain resistance to extinction after extended training on single- and multiple-N-length sequences with a within-Ss design. The direction in the runway that was associated with the multiple- $\mathrm{N}$-length sequence produced greater resistance to extinction than the single-N-length direction. The study also demonstrated that 120 trials in each

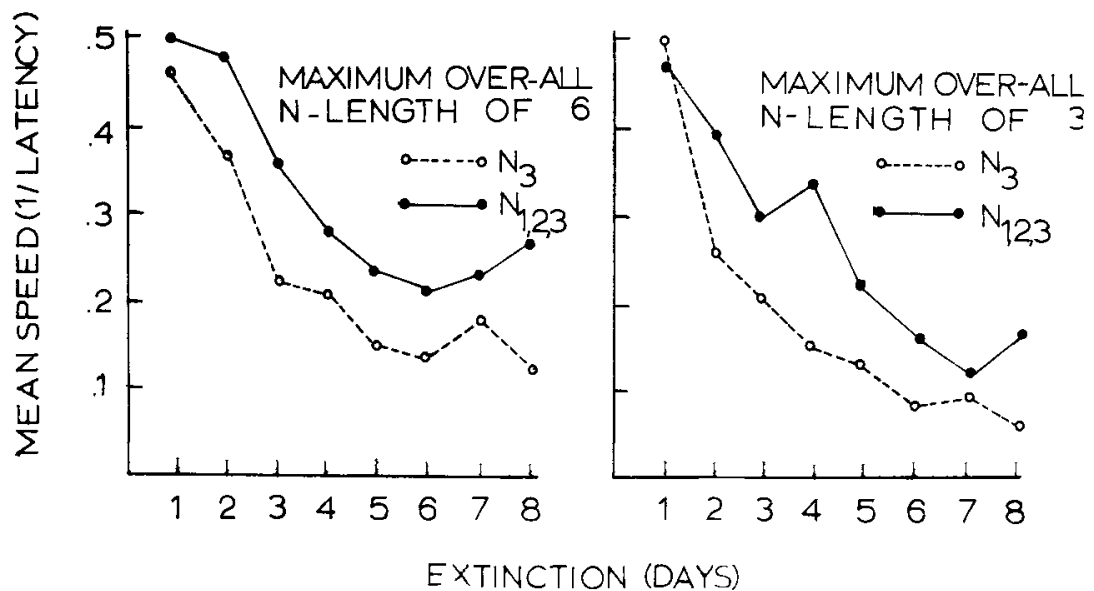

Fig. 1. Resistance to extinction in the two running directions for groups with overall 
direction (the same number of trials Capaldi employed in his between-group design) were enough to constitute "extended" training and produce greater resistance to extinction for the multiple-N-length direction.

Two groups of Ss with different overal] sequences were used to insure that the results of this study and Capaldi's earlier study were determined by the $\mathrm{N}$-lengths being compared rather than by some other variable or variables that might differentiate Capaldi's original single- and multiple-N-length sequences.

It is interesting to attend to the between-groups comparison that can be made. The Ss in one group experienced a maximum $\mathrm{N}$-length of 6 independent of direction, while $S s$ in the other group experienced a maximum $\mathrm{N}$-length of 3 . Both groups experienced their maximum $\mathrm{N}$-length the same number of times. Although the group with the maximum $\mathrm{N}$-length of 3 experienced $\mathrm{N}$-lengths of 1 and 2 more often than the maximum $\mathrm{N}$-length of 6 group, the maximum N-length of 6 group had considerable experience with $\mathrm{N}$-lengths of 4 and 5 ; i.e., the maximum $\mathrm{N}$-length of 6 group experienced the $\mathrm{N}$-lengths of $6,5,4,3,2$, and $1,10,5,0,5$, and 5 times, respectively, while the maximum $\mathrm{N}$-length of 3 group experienced N-lengths of 3,2 , and $1,10,10$, and 70 times, respectively. Thus, the maximum N-length of 6 group: (1) experienced three $\mathrm{N}$-lengths that were greater than those experienced by the maximum N-length of 3 group and (2) experienced as much training on the maximum $\mathrm{N}$-length of 6 as the maximum $\mathrm{N}$-length of 3 group had on the N-length of 3. The maximum N-length of 3 group had more experiences with the short N-lengths of 1 and 2. The greater training on the $\mathrm{N}$-lengths of 1 and 2 for the maximum $\mathrm{N}$-length of 3 group makes an unequivocal statement about resistance to extinction for the two groups impossible. Nonetheless, the sequences suggest that the maximum $\mathrm{N}$-length of 6 group should be considerably more resistant to extinction.

The similar performance that was obtained with both groups can tentatively be explained in terms of the response that was employed. The generic response of running was separated into running in two directions. The within-Ss comparisons suggest that the Ss were making two distinct responses in the two directions. Therefore, it is possible that the $\mathrm{Ss}$ ' behavior was not greatly affected by their overall sequence independent of direction. This would imply that each $\mathrm{S}$ was making two responses and that running independent of direction is not an effective overall response.
The present study demonstrates that sequenced reinforcement effects can be obtained with a within-Ss design. Such a design enables the $\mathrm{E}$ to compare sequences using fewer Ss than needed in a between-groups design. Further, differences obtained with between-group and within-Ss designs can be useful for determining the differences between the two kinds of situations (see Grice, 1967).

\section{REFERENCES}

CAPALDI, E. J., HART, D., \& STANLEY, L. R.
Effect of intertrial reinforcement on the aftereffect of nonreinforcement and resistance to extinction. Journal of Experimental Psychology, 1963, 65, 70-74.

CAPALDl, E. J. Effect of N-length, number of different N-lengths and number of reinforcements on resistance to extinction. Journal of Experimental Psychology, 1964, $68,230-239$.

GRICE, G. R., \& HUNTER, J. J. Stimulus intensity effects depend upon the type of experimental design. Psychologicaî Review, 1967, 71, 247-256.

HEARST, E. Resistance-to-extinction functions in the single organism. Journal of the Experimental Analysis of Behavior, 1961, 4, 133-144.

\title{
Two-cue discrimination learning by pigtail monkeys*
}

\author{
ROSEANN WEBER, University of the Pacific, $†$ Stockton, Calif. 95204 \\ and \\ MARVIN KAMBACK, University of South Dakota, Vermillion, S. Dak. 57069
}

Twelve monkeys were given a discrimination between black vertical and white horizontal rectangles and then given preference tests with original positive and negative stimuli paired with white vertical, black horizontal, white and black diagonal rectangles, and white and black circles. Ss responded more to circles than to other preference stimuli early in preference testing but less later, suggesting a novelty effect. Preference for the reinforced brightness was significantly higher than for the reinforced orientation.

Sutherland \& Holgate (1966) trained rats to discriminate rectangles differing in both brightness and orientation and then gave preference tests with rectangles differing only in brightness or only in orientation. They found consistent negative correlations between appropriate responses to brightness and to orientation, indicating that the more the rat learned about one cue the less it learned about the other. Mumma \& Warren (1968) replicated this study with cats and found a low positive correlation between responses to brightness and orientation. Cats' responses during preference testing were determined by stimulus novelty. Warren \& Warren (1969) conducted a similar experiment with rhesus monkeys. Stimuli used in preference testing, however, were different from those used in training (i.e., black and

*This research was supported by Grant GB-13100X from the National Science Foundation and Grant MH-07147-05 from the National Institute of Mental Health.

tFormerly Roseann Mumma. white circles for brightness and green horizontal and vertical rectangles for orientation) to control for novelty effects. The monkey's preferences for brightness and orientation were significantly negatively correlated, supporting Sutherland and Holgate's interpretation of multiple cue learning.

The present experiment was designed in part to replicate and in part to extend with monkeys Mumma and Warren's experiment with cats, with responsiveness to novelty of primary interest. Similarity between the preference sțimuli and the training stimuli was varied. It was predicted that as the preference stimulus resembled less the training stimulus, $\mathrm{S}$ would respond relatively more to it.

\section{SUBJECTS}

Twelve pigtail monkeys (Macaca nemistrina), weighing from 2.5 to $3.5 \mathrm{~kg}$, were used. They had been adapted to the WGTA and run in a size-color preference experiment. 